\title{
Co-existência de Espécies em Sistemas Presa-predador com Switching
}

S. PALOMINO-BEAN ${ }^{1}$, Depto. de Matemática, UFSC, 88040-900, Florianópolis, SC, Brasil.

A.C.S. VILCARROMERO ${ }^{2}$, J.F.R. FERNANDES, DENSIS/FEEC/UNICAMP, C.P. 6101, 13089-970, Campinas, SP, Brasil.

O. BONATO, IRD, 911 Avenue Agropolis, BP 5045, F-34032, Montpellier, Cedex 1, France.

Resumo. Neste artigo analisamos a co-existência de espécies num sistema de dois níveis tróficos, com duas espécies de presas e uma espécie predadora. Em um primeiro caso consideramos um sistema presa-predador com as espécies interagindo e posteriormente estudamos a inclusão do efeito switching no sistema. O efeito switching é o deslocamento da preferência do predador para um determinado tipo de presa. Em [20] foi estudado esse tipo de comportamento, conseguindo-se a co-existência das espécies em um sistema de dois níveis tróficos com as mesmas espécies. Nele as funções de densidade das presas são de tipo logístico e se inclui um efeito switching usando funções de Tansky no caso mais simples. Dando continuidade ao trabalho, neste artigo analisamos a co-existência das espécies inserindo no modelo a eficiência de consumo das presas pelo predador e considerando o efeito switching no caso geral. A comparação dos resultados obtidos nas simulações numéricas de ambos os sistemas, com e sem switching, mostram que toda vez que é considerado o efeito switching o sistema se estabiliza, mantendo assim a co-existência das espécies.

\section{Introdução}

O modelo clássico de Lotka-Volterra para multi-espécies tem gerado muitas pesquisas no estudo das interações de populações do tipo presa-predador. Uma das linhas de estudo considera o fato de alguns predadores polífagos deslocarem sua preferência para uma determinada presa, dependendo da freqüência relativa desta. Um predador que se alimenta de várias espécies de presa não ataca todos os tipos indiscriminadamente. Quando um tipo de presa torna-se escasso no meio ambiente, o predador pode parar de procurar por esta espécie totalmente e começar a caçar uma outra espécie mais abundante [11]. Este tipo de comportamento do predador

\footnotetext{
${ }^{1}$ palomino@mtm.ufsc.br

2 angelavilcarromero@yahoo.com.br
} 
é conhecido como switching, ou deslocamento da preferência do predador para um determinado tipo de presa agindo como uma influência estabilizadora na interação das espécies. O comportamento dinâmico e as propriedades switching em sistemas presa-predador foram estudados por Murdoch [8], Tansky [16], Mukherjee [7], Matsuda [5] entre outros. Estes e outros estudos realizados com o efeito switching do predador mostraram que este pode estabilizar o sistema como um todo e garantir a co-existência e a competição permanente das espécies ([11]-[5],[7]). Isto está baseado em fatos empíricos, experimentais e teóricos realizados por muitos autores ([2],[8]-[14],[1]). Em particular Matsuda [5] considera que efeitos switching podem ser determinados por comportamentos estratégicos do predador, denominando-os como Evolutionary Stable Strategies, ESS. Mukherjee [6, 7] estuda sistemas mais complexos adicionando, ao estudo de estabilidade assintótica (global e local), o efeito switching do predador no modelo. Já Pelletier [14] faz um estudo dos modelos de multi-espécies de Lotka-Volterra e, utilizando métodos numéricos, afirma que sistemas mais complexos são mais estáveis quando considerado o efeito switching do predador.

À exceção de Mukherjee [7], todos os trabalhos anteriormente citados consideram que a função de densidade das presas tem um crescimento exponencial. Uma forma mais realística é considerar um crescimento logístico como feito em Vilcarromero [20], onde se mostra a co-existência das espécies do sistema presa-predador incluindo o switching do predador caracterizado pelas funções de Tansky [16] (no caso mais simples) atuando em cada uma das funções de densidade que descrevem o comportamento das presas. Dando continuidade ao trabalho desenvolvido em [20], este artigo além de considerar o comportamento logístico das presas leva em consideração o caso geral das funções de Tansky para o efeito switching e a resposta numérica do predador ao consumir sua presa, conforme será visto na formulação do problema na seção 2. A seção 3 descreve a metodologia utilizada e na seção 4 é feito o estudo de estabilidade apresentando os resultados. Por último, nas conclusões, são discutidos os resultados deste artigo.

\section{Formulação do Problema}

Usando o modelo geral de Lotka-Volterra para multi-espécies e dando continuidade ao trabalho desenvolvido em [20], nesta seção formulamos o problema a ser tratado considerando um sistema de dois níveis tróficos com duas presas e um predador.

Lotka [3] e Volterra [21] estabeleceram um modelo clássico dado por um conjunto de equações diferencias ordinárias da forma:

$$
\begin{aligned}
& \dot{X}=F(X)-G(X, Y) \\
& \dot{Y}=U(G(X, Y))-V(Y),
\end{aligned}
$$

onde $X=\left[x_{1}, \cdots, x_{n}\right]^{\prime}$ e $Y=\left[y_{1}, \cdots, y_{n}\right]^{\prime}$ são, respectivamente, as densidades das presas e dos predadores. As funções $F=\left[f_{1}, \cdots, f_{n}\right]^{\prime}, \quad G=\left[g_{1}, \cdots, g_{n}\right]^{\prime}, \quad U=$ $\left[u_{1}, \cdots, u_{n}\right]^{\prime}$ e $V=\left[v_{1}, \cdots, v_{n}\right]^{\prime}$ representam a reprodução das presas (crescimento intrínseco), mortalidade da presa devido a predação, reprodução do predador e mortalidade do predador, respectivamente. Com intuito de utilizar um modelo 
mais realístico, em [20] o problema foi modelado usando as funções de Tansky [16] (no caso mais simples) e um crescimento logístico na população das presas da forma:

$$
f_{i}\left(x_{i}\right)=r_{i} x_{i}\left(1-\frac{x_{i}}{k_{i}}\right)
$$

onde $r_{i}$ e $k_{i}$ são constantes positivas que indicam a taxa de reprodução da presa $i$ e a capacidade de suporte do meio para a presa $i,(i=1,2)$ respectivamente.

Neste trabalho consideramos uma população com dois níveis tróficos e três espécies: duas presas e um predador. Portanto, consideramos um crescimento logístico para as presas como em (2.2), modificamos a função de densidade do predador $^{3}$ e incluímos um efeito switching usando as funções de Tansky [16] na sua forma geral.

Para facilitar a notação do sistema de equações diferenciais (2.1), consideramos as duas primeiras espécies como sendo as presas, $x_{1}$ e $x_{2}$, e a última espécie como sendo a do predador, $y_{1}=x_{3}$. Assim, a variável predatória será colocada no vetor de densidade das espécies $X$, isto é, $X=\left[x_{1}, x_{2}, x_{3}\right]^{\prime}$.

Partimos do suposto de que não há competição entre as presas e que elas não interagem com presas de outras espécies.

O problema é formulado através de dois modelos. O primeiro modelo será formulado sem considerar o switching do predador e no segundo modelo o switching será incluído.

O modelo sem o switching do predador é o modelo clássico de Lotka-Volterra dado por:

$$
\begin{aligned}
& \dot{x}_{1}=\left(r_{1}-\frac{r_{1}}{k_{1}} x_{1}-a_{1} x_{3}\right) x_{1} \\
& \dot{x}_{2}=\left(r_{2}-\frac{r_{2}}{k_{2}} x_{2}-a_{2} x_{3}\right) x_{2} \\
& \dot{x}_{3}=\left(-r_{3}+c_{1} a_{1} x_{1}+c_{2} a_{2} x_{2}\right) x_{3}
\end{aligned}
$$

No modelo, $r_{i}$ é a taxa de crescimento intrínseco da presa $i,(i=1,2) ; k_{i}$ é a capacidade de suporte do meio para a presa $i,(i=1,2) ; r_{3}$ é a taxa de mortalidade do predador, $a_{i}$ é o coeficiente da eficiência de procura do predador em relação a presa $i,(i=1,2)$, e $c_{i}$ é a resposta numérica (taxa de reprodução) do predador ao consumir a presa $i,(i=1,2)$. Todos estes valores utilizados são considerados constantes.

Modelos com switching têm efeito na estabilidade das populações, conforme verificado por fatos empíricos, experimentais e teóricos dos autores citados anteriormente. O modelo com switching é descrito por:

$$
\begin{aligned}
& \dot{x}_{1}=\left(r_{1}-\frac{r_{1}}{k_{1}} x_{1}-A_{1}\left(x_{1}, x_{2}\right) x_{3}\right) x_{1} \\
& \dot{x}_{2}=\left(r_{2}-\frac{r_{2}}{k_{2}} x_{2}-A_{2}\left(x_{1}, x_{2}\right) x_{3}\right) x_{2} \\
& \dot{x}_{3}=\left(-r_{3}+c_{1} A_{1}\left(x_{1}, x_{2}\right) x_{1}+c_{2} A_{2}\left(x_{1}, x_{2}\right) x_{2}\right) x_{3},
\end{aligned}
$$

\footnotetext{
${ }^{3}$ Isto é, na taxa de variação do predador consideraremos a resposta numérica do predador ao consumir suas presas.
} 
onde $r_{3}, r_{i}, k_{i}, c_{i} \quad(i=1,2)$ são descritos como em (2.3). $A_{1}$ e $A_{2}$ são consideradas como as funções de Tansky [5] que na sua forma geral estão dadas por:

$$
\begin{aligned}
& A_{1}\left(x_{1}, x_{2}\right)=a_{1} /\left(1+\left(\frac{x_{2}}{x_{1}}\right)^{n}\right) \\
& A_{2}\left(x_{1}, x_{2}\right)=a_{2} /\left(1+\left(\frac{x_{1}}{x_{2}}\right)^{n}\right),
\end{aligned}
$$

onde $a_{i}$ é descrita como em (2.3) e a potência $n$ é denominada intensidade do switching ${ }^{4}$, fornecendo uma formulação geral ao modelo em estudo neste trabalho (o caso mais simples dessas funções, $n=1$, foi usado em [20]). As funções $A_{1}, A_{2}$ representão as interações não constantes entre o predador e suas presas variando conforme o valor dado por $n$. A influência desse valor dado é explicada logo a seguir.

Considere que essas funções são representadas graficamente pela superfície definida pelos pontos $\left(x_{1}, x_{2}, A_{i}\left(x_{1}, x_{2}\right)\right)$. Quando o valor de $n$ cresce, a parte inferior da superfície se aproxima do plano $z=0$ e o topo dela se aproxima do plano $z=d \quad$ ( $d$ uma constante positiva). Isto é, à medida que $n$ cresce as funções são representadas por sigmóides espaciais, propriedade característica do mecanismo de switching $[11,16]$. Observe essas variações nos gráficos da Figura 1, para valores de $n$ iguais a 1 e 10 , respectivamente.

Vejamos o que acontece quando $A_{1}$ e $A_{2}$ são consideradas no modelo em estudo. Quando fornecemos valores $\left(x_{1}, x_{2}\right)$ no domínio (quadrado de lado 5 delimitado pelo gráfico), avaliamos os pares ordenados abaixo da diagonal dados pelos pontos $(0,0)$ e $(5,5)$. Neste caso, a ação da função $A_{1}$ é praticamente nula. No entanto, a função $A_{2}$ tem plena participação. Nos pontos acima dessa diagonal ocorre um processo totalmente oposto, é a função $A_{1}$ que possui plena participação. Desse modo, a função $A_{i}$, para cada $i(i=1,2)$, terá mais influência na obtenção da trajetória solução (o que será visto depois) quanto maior for o valor dado a $n$. Daí o porque do nome switching para a ação do predador.

\section{Metodologia}

Para determinar a estabilidade dos sistemas com e sem switching em primeiro lugar são obtidos os possíveis pontos de equilíbrio não triviais fazendo uso do método de Newton - Raphson. Depois, usando a matriz do sistema linearizado, analisamos a estabilidade dos pontos de equilíbrio obtidos usando o método de Routh-Hurwitz. Por último, escolhendo a condição inicial (tomando o ponto de equilibrio como referência) encontramos a solução do sistema através do método de Runge-Kutta.

Consideremos em primeiro lugar, o modelo sem ação do efeito switching. As equações que governam o modelo foram dadas em (2.3) e, como obtido em [20], leva à extinção de uma das espécies.

No segundo caso é considerado o efeito switching no sistema. Considerando-se as funções fornecidas na equação (2.5), o sistema vem dado por:

\footnotetext{
${ }^{4}$ Observe que, sem perda de generalidade, podemos considerar uma intensidade nula ao efeito switching, isto é, poderemos reproduzir o modelo clássico de Lotka-Volterra ao fazer as funções $A_{1}, A_{2}$ como sendo iguais as constantes $a_{1}, a_{2}$ da equação (2.3), respectivamente.
} 

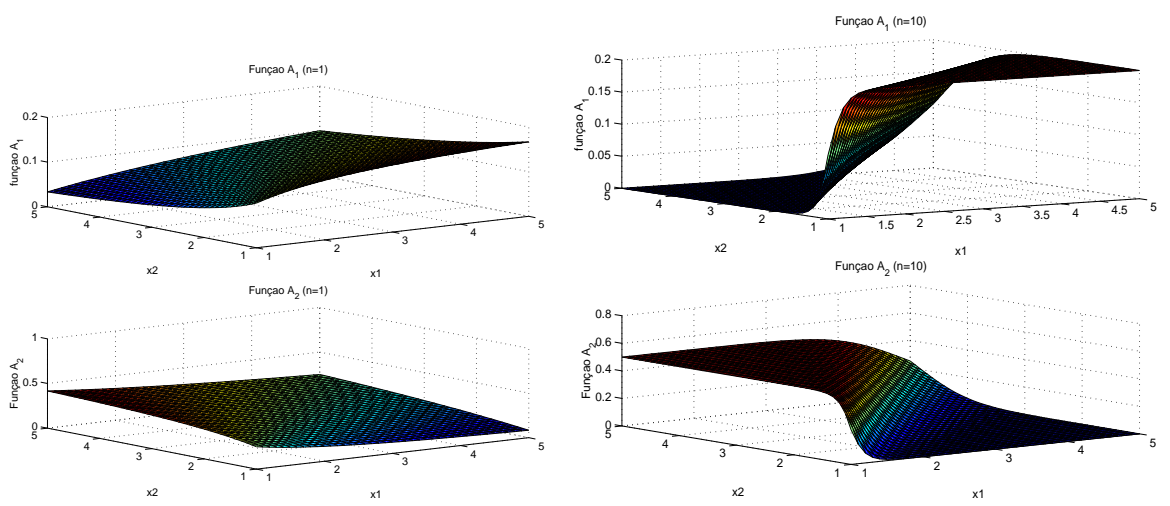

Figura 1: Funções switching nos casos $n=1$ (à esquerda) e $n=10$ (à direita) com parâmetros $a_{1}=0.2, a_{2}=0.5$.

$$
\begin{aligned}
& \dot{x}_{1}=\left(r_{1}-\frac{r_{1}}{k_{1}} x_{1}-a_{1} \frac{x_{1}{ }^{n}}{x_{1}^{n}+x_{2}^{n}} x_{3}\right) x_{1} \\
& \dot{x}_{2}=\left(r_{2}-\frac{r_{2}}{k_{2}} x_{2}-a_{2} \frac{x_{2}{ }^{n}}{x_{1}^{n}+x_{2}{ }^{n}} x_{3}\right) x_{2} \\
& \dot{x}_{3}=\left(-r_{3}+\frac{c_{1} a_{1} x_{1}{ }^{n+1}}{x_{1}{ }^{n}+x_{2}^{n}}+\frac{c_{2} a_{2} x_{2}{ }^{n+1}}{x_{1}{ }^{n}+x_{2}^{n}}\right) x_{3}
\end{aligned}
$$

e os parâmetros definidos como na equação (2.3) e (2.4).

Toda vez que o predador se alimenta de alguma presa, a taxa de crescimento da população do predador e a taxa de mortalidade da presa escolhida são modificados pelo mesmo fator, assim os termos de interação relativa aparecem em ambos os sistemas (2.3) e (3.1).

Na seguinte seção são apresentados os resultados obtidos das simulações numéricas dos modelos.

\section{Resultados}

Simulando e comparando os modelos dados em (2.3) e (3.1), para diversos valores de $n$ e o mesmo conjunto de parâmetros, pode-se observar a influência estabilizadora do efeito switching do predador. O lado esquerdo da Fig. 2 mostra as trajetórias de cada uma das espécies do modelo sem o switching do predador, dado pela equação (2.3). Para diversos conjuntos dos parâmetros $r_{i}, k_{i}$ e $a_{i}$ escolhidos, observou-se a instabilidade do ponto de equilíbrio não trivial, em que uma das espécies, a espécie $x_{2}$, vai para a extinção (Fig.2 (a) á esquerda) num intervalo de tempo menor do que 20 unidades de tempo. Na mesma figura (Fig.2 (b, c, d) à esquerda) se exibe o retrato de fase e os planos de fase desse sistema. Temos o ponto de equilíbrio não trivial $\left(x_{1}{ }^{*}, x_{2}{ }^{*}, x_{3}{ }^{*}\right)$ instável cujas espécies projetadas no plano de fase $x_{2} x_{3}$, evoluem numa trajetória em que $x_{2}$ vai para extinção (Fig. 2 (d) à esquerda). Já 
na projeção do retrato de fase do sistema no plano $x_{1} x_{3}$, a trajetória é de tipo ciclo limite (Fig.2 (c) à esquerda ).
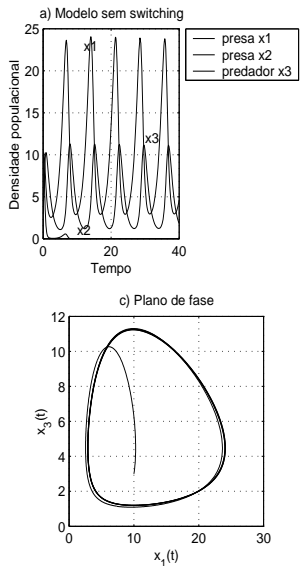
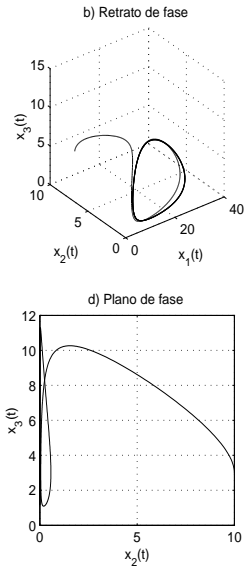
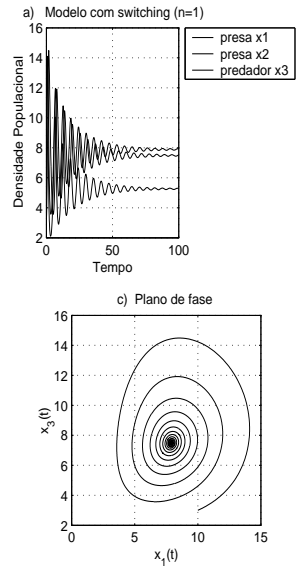
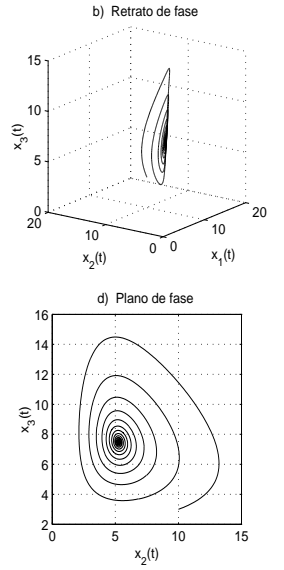

Figura 2: À esquerda: Modelo sem switching: a) Densidade populacional das populações $x_{i}, i=1,2,3$ como uma função do tempo. b) Retrato de fase. c) Plano de fase das espécies $x_{1}, x_{3}$ e o seu ciclo limite. d) Plano de fase das espécies $x_{2}, x_{3}$, mostrando a instabilidade. À direita: Modelo com switching $(\mathrm{n}=1)$. a) Evolução da trajetória de cada espécie no tempo, b) O retrato de fase, c) e d) Os planos de fase e estabilidade assintótica. Constantes consideradas no modelo: $r_{1}=0.9, r_{2}=1,5, r_{3}=1.0, k_{1}=k_{2}=5000, a_{1}=0.2, a_{2}=$ $0.5, c_{1}=0.5, c_{2}=0.5$. Ponto de co-existência: $x_{1}{ }^{*}=7,93, x_{2}{ }^{*}=5,29, x_{3}{ }^{*}=7,52$.

Considerando-se os mesmos parâmetros e as mesmas condições iniciais do modelo (2.3), simulamos numéricamente os resultados do modelo com o switching do predador dado pela equação (3.1). Nas Fig. (2 à direita), (3) e (4), podemos observar a evolução das trajetórias para cada uma das espécies em que $n$ é igual a 1, 5, 20 e 100. Neste caso, também temos a estabilidade do ponto de equilíbrio não trivial ou ponto de co-existência das espécies (Fig. 2 b ( à direita) e Fig. 3 b), confirmando esses resultados nos planos de fase das figuras Fig. 2 (c, d à direita) e Fig. 3 (c, d ). Há uma diferença de escalas quando comparamos com a Fig. 2 (a) pelo fato de uma das espécies ir à extinção quando $t$ é menor a 20 unidades de tempo, enquanto que as outras permanecem oscilantes no tempo. Já em ambos casos das Fig. (2 à direita), Fig. 3 e Fig. 4, as trajetórias estabilizam num tempo maior a 80 unidades para atingir a co-existência. Também, pode-se observar que embora o tempo percorrido para atingir estabilidade é maior quanto maior for o valor de $n$, esses tempos são menores quando comparados ao caso $n=1$.

\section{Conclusões}

Neste trabalho foi analisada uma generalização do modelo clássico Lotka-Volterra com e sem o acréscimo de um termo que caracteriza o efeito switching do predador. Ambos modelos foram comparados para estudar o comportamento de uma 

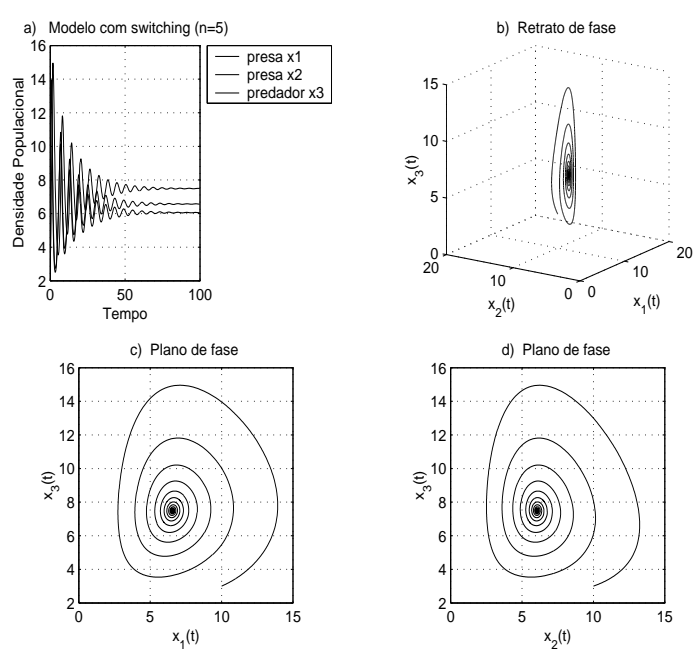

Figura 3: Quando $n=5$ o comportamento das trajetórias são similares no retrato e nos planos de fase. Neste caso, a estabilidade assintótica acontece quando as espécies assumem os valores $x_{1}^{*}=6,56, x_{2}^{*}=6,05, x_{3}^{*}=7,49$.

população de duas presas e um predador.

Em [18, 19], foi analisado analiticamente o efeito da variação da resposta funcional do modelo presa-predador do tipo Rosenzweig-MacArthur. Em [12], usamos inequações matriciais lineares para estudar a estabilidade local de sistemas chaveados (ou switching systems) utilizando duas aproximações diferentes, e em [20] conseguimos a co-existência das espécies num sistema com duas presas e um predador, com o efeito switching, usando as funções de Tansky no caso mais simples ${ }^{5}$.

Considerando-se o modelo presa-predador sem incluir o efeito switching do predador, foi observado neste artigo que, para diferentes conjuntos de parâmetros, sempre uma das espécies de presa vai à extinção. Entretanto, se incluímos as funções switching no modelo, as trajetórias convergem assintoticamente para o seu ponto de equilíbrio. Isso foi observado nos planos de fase das Fig. 3 e $4(n>1)$. Tomando diferentes valores para $n$, constatamos (Fig. 4) que quanto maior o valor de $n$ mais rápida a convergência do sistema para o seu ponto de equilíbrio. Por tanto, podemos concluir que ao considerarmos o caso geral do switching do predador estabilizamos o sistema como um todo garantindo a co-existência permanente das espécies.

Os resultados numéricos obtidos neste trabalho, assim como em [20], são a continuidade de uma nova linha de pesquisa na qual estamos trabalhando: o switching do predador em sistemas de populações. Pretendemos continuar o estudo analisando a estabilidade do ponto de equilíbrio usando outras técnicas como desenvolvidas em [12] e [13] e estender a pesquisa considerando o efeito switching em populações [15],

\footnotetext{
${ }^{5}$ Quando $n=1$, as funções de Tansky são dadas por $A_{i}\left(x_{1}, x_{2}\right)=a_{i} \frac{x_{i}}{x_{1}+x_{2}}$.
} 

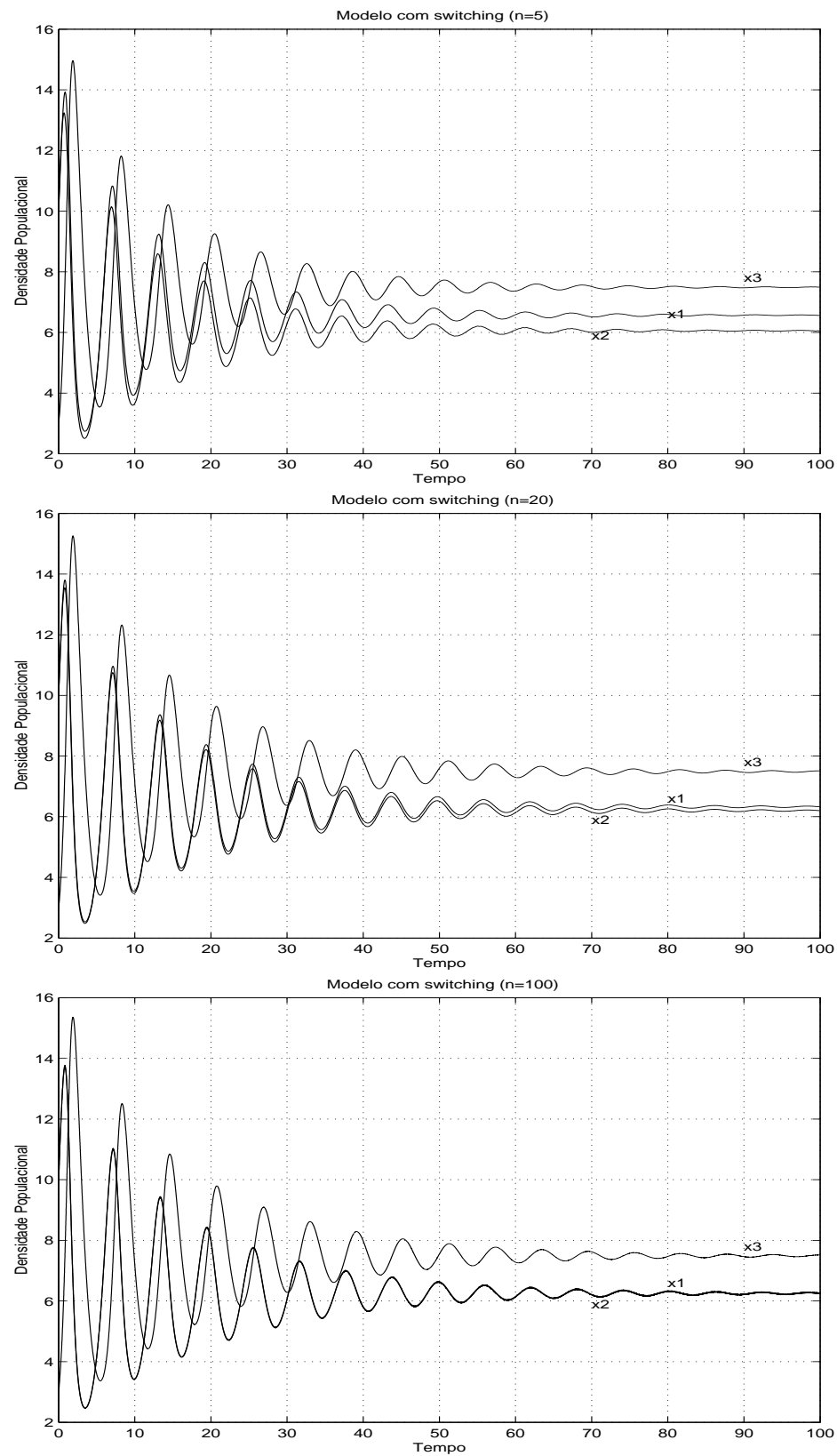

Figura 4: Evolução das trajetórias nos casos $n=5,20$ e 100. Quanto maior for o valor de $n$, a estabilidade é atingida em tempos maiores, porém próximos, e a co-existência permanece. Todos esses níveis de tempo são menores quando comparados ao caso $n=1$. Para valores de $n$ entre 20 e 100 não há muita diferença entre os valores dos pontos fixos obtidos para conseguir a estabilidade assintótica: $x_{1}^{*}=6,3, x_{2}^{*}=6,2, x_{3}^{*}=7.5$. 
assim como associar uma função de controle aos modelos em estudo.

\title{
Agradecimentos
}

Os autores expressam seus agradecimentos aos colegas do Dpto. de Matemática, Dr. F. Bazán e Dr. M. Braitt, e aos revisores anônimos pelas importantes colocações que ajudaram na melhoria deste artigo.

\begin{abstract}
This article is a continuation of a previous work [20] which includes a switching function in a prey -predator system of two prey and one predator with two trophic levels. A geral Tansky switching function is used and it is verified that independent of the value of the function intensity, the species go to asymptotic stability, that is the coexistence remains.
\end{abstract}

\section{Referências}

[1] P.A. Abrams, H. Matsuda, Positive indirect effects between prey species that share predators, Ecology, $\mathbf{7 7}$ (1996), 610.

[2] E. Kuno, Principles of predator-prey interaction in theoretical, experimental, and natural population systems, Advances in Ecological Research, 16 (1987), 249-337.

[3] A.J. Lotka, "Elements of Physical Biology", Williams and Wilkins, Baltimore, 1925.

[4] H. Matsuda, Evolutionary stable strategies for predator switching, J. Theor. Biol., 115 (1985), 351-366.

[5] H. Matsuda, K. Kawasaki, N. Shigesada, E. Teramoto, L.M. Ricciardi, Switching effect on the stability of the prey-predator system with three trophic levels, J. Theor. Biol., 122 (1986), 251-262.

[6] D. Mukherjee, A.B. Roy, Global stability of prey-predator systems with predatory switching, BioSystems, 27, No. 3 (1992), 171-178.

[7] D. Mukherjee, A.B. Roy, On local(ly) ESS of a pair of prey-predator system with predatory switching, Math. Biosciences, 151 (1998), 165-177.

[8] W.W. Murdoch, Switching in general predators: experiments on predator specificity and stability of prey populations, Ecol. Monographs, 39, No. 4 (1969), 335-354.

[9] W.W. Murdoch, S. Avery, M.E.B. Smyth, Switching in predatory fish, Ecology, 56, No. 5 (1975), 1094-1105.

[10] W.W. Murdoch, J.R. Marks, Predation by coccinellid beetles: Experiments on switching, Ecology, 54, No. 1 (1973), 160-167. 
[11] A. Oaten, W.W. Murdoch, Switching, functional response, and stability in predator-prey systems, The American Naturalist, 109, No. 967 (1975), 299318 .

[12] S. Palomino-Bean, J.C. Geromel, A.Trofino, J.E.R. Cury, Comparative methods for computation of stability regions of a class of non-linear systems. Em "Anais da 1a. Escola Brasileira de Aplicações em Dinâmica e Controle", São Carlos, SP, Brazil, 2001.

[13] S. Palomino-Bean, Abordagem Qr para estabilidade regional de sistemas chaveados, "Seleta do XXV CNMAC" (E.X.L. de Andrade, G.N. Silva e A. Sri Ranga, eds.), Tendências em Matemática Aplicada e Computacional, Vol. 4, No. 2, 167-176, SBMAC, 2003.

[14] J.D. Pelletier, Are large complex ecosystems more unstable? A theoretical reassessment with predator switching, Math. Biosciences, 163 (2000), 91-96.

[15] M. Saleem, A.K. Tripathi, A.H. Sadiyal, Coexistence of species in a defensive switching model, Math. Biosciencies, 181 (2003), 145-164.

[16] M. Tansky, Switching effect in prey-predator system, J. Theor. Biol., 70 (1978), 263-271.

[17] E. Teramoto, K. Kawiasaki, N. Shigesada, Switching effect of predation on competitive prey species, J. Theor. Biol., 79 (1979), 303-315.

[18] A.C.S. Vilcarromero, O. Bonato, Modelagem matemática e simulação das interações entre o ácaro verde-predador nativo-predador exótico no agroecossistema da mandioca. Em "Anais do XXII CNMAC", Santos, SP, Brazil, 1999.

[19] A.C.S. Vilcarromero, O. Bonato, J.F.R. Fernandes, Stability analysis in a classical predator-prey model: Example of cassava mites system. Em "Abstracts of XXI International Congress of Entomology", Foz do Iguaçu, PR, Brazil, 2000.

[20] A.C.S. Vilcarromero, S. Palomino-Bean, O. Bonato, J.F.R. Fernandes, Switching behavior and stability in predator-prey systems. Em "Anais do X Congresso Latinoamericano de Biomatemática, X ALAB", Campinas, SP. Brazil, 2001.

[21] V. Volterra, "Variazioni e fluttuazioni dei numero di individui in specie animali conviventi", Mem. Acad. Lincei, 2 (1926), 31-113. (English translation in Chapman, R. N. Animal Ecology, McGraw-Hill, New York, 1931). 